\title{
An approach for determining and measuring network hierarchy applied to comparing the phosphorylome and the regulome
}

\author{
Chao Cheng ${ }^{1,2,3^{*}}$, Erik Andrews ${ }^{1}$, Koon-Kiu Yan ${ }^{4}$, Matthew Ung ${ }^{1}$, Daifeng Wang ${ }^{4}$ and Mark Gerstein ${ }^{4,5,6^{*}}$
}

\begin{abstract}
Many biological networks naturally form a hierarchy with a preponderance of downward information flow. In this study, we define a score to quantify the degree of hierarchy in a network and develop a simulated-annealing algorithm to maximize the hierarchical score globally over a network. We apply our algorithm to determine the hierarchical structure of the phosphorylome in detail and investigate the correlation between its hierarchy and kinase properties. We also compare it to the regulatory network, finding that the phosphorylome is more hierarchical than the regulome.
\end{abstract}

\section{Background}

Networks have been used as universal frameworks to represent many complex systems including the World Wide Web [1], social interactions [2], literature citation relationships [3], and biological processes [4-6]. Based on the attributes of edges, networks can be subdivided into two categories: undirected and directed. In an undirected network there is no distinction between the two vertices associated with each edge, whereas in a directed network all edges are directed from one vertex to another. The asymmetric nature of edges in a directed network causes topological differences of nodes, resulting in a hierarchical structure: some function as top regulators, while others function as downstream effectors.

Owning to the development of large-scale experimental techniques, many biological networks have been produced. These include protein-protein interaction networks and genetic interaction networks [7-12]. Among them, the gene regulatory network (referred to as the regulome) and the protein phosphorylation network (referred to as the phosphorylome) are two of the best-studied directed networks $[10,11]$. The regulome captures the transcriptional regulatory interactions of transcription factors (TFs) with their target genes. The techniques to systematically

\footnotetext{
* Correspondence: chao.cheng@dartmouth.edu; mark.gerstein@yale.edu ${ }^{1}$ Department of Genetics, Geisel School of Medicine at Dartmouth, Hanover, New Hampshire, USA

${ }^{4}$ Program in Computational Biology and Bioinformatics, Yale University, 260 Whitney Avenue, New Haven, CT 06520, USA

Full list of author information is available at the end of the article
}

identify TF-DNA interactions include the bacterial onehybrid system [13], the yeast one-hybrid system [14], and chromatin immunoprecipitation followed by microarray (ChIP-chip) [15] or parallel sequencing (ChIP-seq) [16]. In particular, ChIP-chip and ChIP-seq have been used to determine the target genes of a large number of TFs in recent years, and will produce more data in the near future. In particular, in yeast Harbison et al. have performed ChIP-chip experiments to identify target genes of 203 proteins, which represent nearly all of the DNA-binding transcriptional regulators encoded in the yeast genome [10]. In human, the Encyclopedia of DNA Elements (ENCODE) project has determined the genomic binding sites of more than 120 TFs [17]. Meanwhile, the interactions between kinases, phosphotases, and their substrates can be identified by protein chip [11] or mass spectrometry [18]. The latter technology is capable of providing precise phosphorylation sites. In particular, Ptacek et al. has determined the in vitro substrates recognized by most yeast protein kinases [11]. The availability of these datasets enables us to construct regulomes and phosphorylomes and investigate the regulatory mechanisms of TFs and kinases on a systems level.

Since the regulome and phosphorylome are directed networks, it is of particular interest to examine whether they harbor a hierarchical structure (TF/kinase nodes function at different levels) and, if so, how that hierarchy is organized. Particularly, we have previously investigated the rewiring of the regulomes in E. coli and S. cerevisiae, and found that hierarchy, rather than connectivity, better 
reflects the importance of regulators [19]. For the regulomes, the hierarchy properties have been explored in several studies [17,20-23]. In these studies, the authors inferred the hierarchical structure of regulomes and examined the correlation between the hierarchy and TF features. For example, Jothi et al. demonstrated that toplevel TFs in the yeast regulome are more likely to be essential and are more conserved across species [21]. These studies provide critical insights into the regulatory mechanisms of TFs during transcription regulation. On the other hand, the phosphorylation network has not been investigated from a hierarchical perspective.

Several algorithms have been proposed to infer the hierarchical structure of directed networks [20,21,24-27], including leaf-removal, breadth-first-search (BFS) and vertex sort (VS) methods. These algorithms have been applied to the regulome and revealed new insights on hierarchical organization of TFs during transcriptional regulation. Despite their effectiveness, they have several limitations and do not address some important issues about hierarchical networks. The leaf-removal algorithm determines the hierarchical structure by removing leaves iteratively, and as a consequence it cannot be applied to a directed network with cycles. Similarly, for networks with cycles the BFS method has to break cycles before assigning hierarchical levels to nodes [20]. In addition, these two methods do not allow any ambiguity in positioning a node among hierarchical level - a feature that is often the case in many networks. The VS algorithm proposed by Jothi et al. is capable of overcoming these shortcomings. However, it can only assign ambiguous nodes to an interval of potential levels without providing the probability of them to be at each level [21].

Moreover, these methods have not addressed several important questions related to hierarchical networks: How to quantify the degree of hierarchical structure for a given network? How to estimate significance of hierarchical structure of a directed network? How to compare the degree of hierarchy of two directed networks? Importantly, can every node be assigned to a specific level with the same confidence? If not, how can we know which nodes are more confident than the others? For those ambiguous nodes, what are the probabilities of them to be assigned to each level?

The degree of hierarchy for a given for a given network is not well-defined concept. Ispolatov et al. [27] introduced the idea of dominant direction by minimizing the number of feedback links. While it is a proxy of hierarchical structure to a certain extent, the method does not provide a rigorous statistical confidence. Here, we define a metric to quantify the degree of hierarchy for a given hierarchical network, and then propose a new method called hierarchical score maximization (HSM) to infer the hierarchy of a directed network. First, we apply the algorithm to a military command network which possesses a perfect hierarchical structure. The results demonstrate its effectiveness in precisely determining the network's hierarchy. Second, we apply the algorithm to eight directed networks including biological networks, social networks, and ecological networks. We compare these networks in terms of their degrees of hierarchy and the results suggest that phosphorylomes are more hierarchical than transcriptional regulatory networks. Third, we compare the hierarchical structure of the yeast regulome determined using the HSM algorithm with those from previous algorithms. Finally, we investigate the hierarchical structure of the yeast phosphorylome in detail and relate kinases in different levels with different genomic features.

\section{Results}

\section{Construction of hierarchy by simulated annealing}

To infer the hierarchical structure of a directed network, we start by defining a score to quantify the degree of hierarchy. For a network with a specified hierarchical topology (that is, every node is assigned to a specific hierarchy level), there are in general three types of edges: downward interactions (pointing from higher-level to lower-level nodes), upward interactions (pointing from lower-level to higher -level nodes), and horizontal interactions (between nodes in the same level). We thus define the hierarchy score (HS) as the ratio of the number of downward interactions $\left(\mathrm{N}_{\mathrm{d}}\right)$ to the number upward interactions $\left(\mathrm{N}_{\mathrm{u}}\right)$ balanced by the number of horizontal interactions $\left(\mathrm{N}_{\mathrm{h}}\right)$ (see 'Materials and methods' for details) (Figure 1A). Based on this definition, we infer the hierarchical structure of a directed network as the one that achieves the maximum hierarchy score. Specifically, a simulated annealing algorithm is used to continuously adjust the hierarchical structure until the hierarchy score is maximized (Figure 1B). Since HS will increase as the number of levels is increased, the HS for two hierarchical networks with different numbers of levels are in general not directly comparable. To address this issue, we therefore elaborate HS into a new metric called the corrected hierarchy score (CHS), which quantifies the enrichment in downward flow relative to expectation (see 'Materials and methods' for details). Finally, we define a $P$ value for how likely one would get such a hierarchical structure randomly.

In principle, the optimum hierarchical structure for a directed network may not be unique due to the existence of loops. Some nodes can be assigned to different levels without significant change of hierarchy score. For this reason, it is more reasonable and more informative to represent the hierarchical structure as a probabilistic model, in which a node may be assigned to multiple levels with different probabilities. To estimate these 


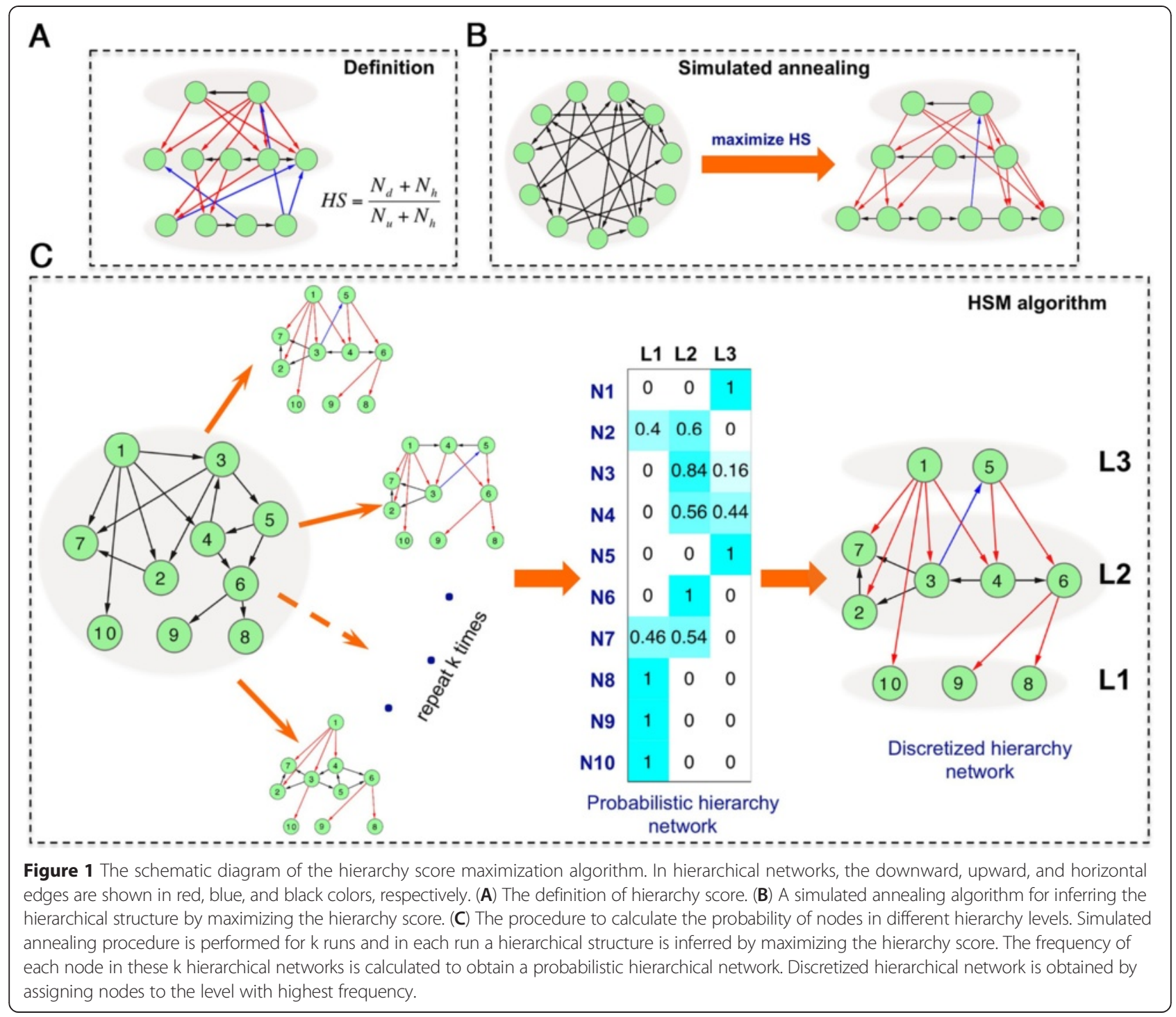

probabilities, for a directed network we performed the simulated annealing procedure 1,000 times $(\mathrm{k}=1,000)$; aggregating the results from each run gives rise to probabilistic assignments to the different levels (Figure 1C). Accordingly, we define a score called the probabilistic hierarchy score (PHS) to more accurately quantify the hierarchy underlying a directed network (see 'Materials and methods' for details). Typically, most of the nodes have a favored level to which the node is assigned with a significantly higher probability than the other levels. We thus can obtain a determined hierarchical structure by assigning each node to its most likely level (Figure 1C).

\section{Application of the HSM algorithm to a military command network}

To show the effectiveness of our method we apply it to a military command network, which we know is a directed acyclic graph (DAG) with a perfect hierarchical structure. Since there are no loops in the network, the hierarchy levels of each node can be deterministically assigned (Figure 2A). We then apply the HSM algorithm to the network, specifying different number of levels $\mathrm{L}=$ $2,3, \ldots, 8$. As shown in Figure 2B, the hierarchical structure is precisely inferred when the correct number of levels $(L=5)$ is specified. All of the nodes are assigned to the right levels with $100 \%$ certainty. Meanwhile the largest HS, CHS, and PHS were obtained when $\mathrm{L}$ is set to 5 . In practice, we do not have the prior knowledge about the number of hierarchical levels. To determine the number of levels one can try a range of different $\mathrm{L}$ values and then set $L$ to a 'saturating value' so that further increase of $\mathrm{L}$ results in no or little increase in $\mathrm{CHS}$ and PHS.

To show that the HS can quantify the degree of hierarchy of a directed network we perturb the original network in Figure 1A by introducing a number of upward 


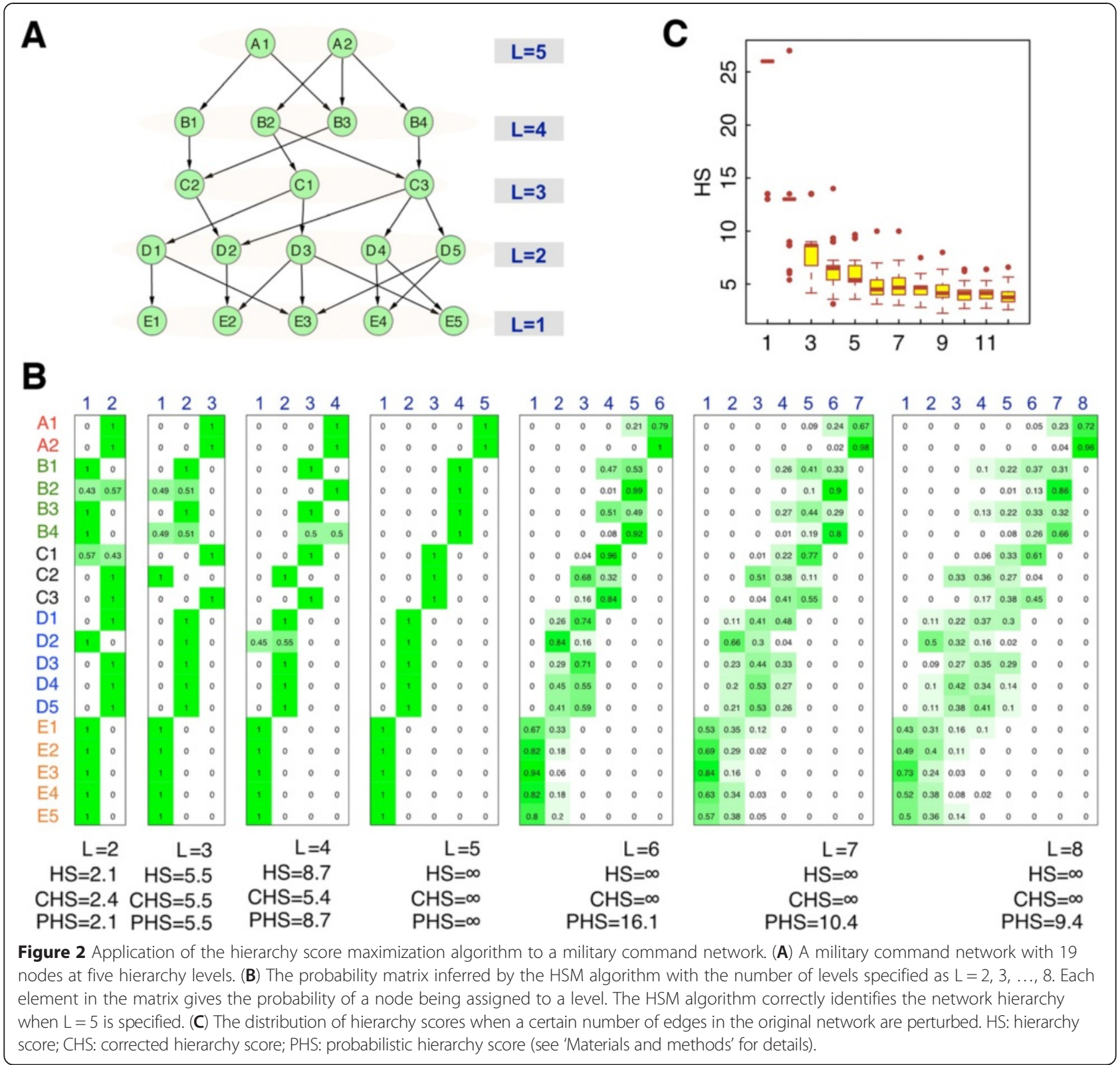

edges. We randomly introduce a number of upward edges (n) to perturb the hierarchical structure of the network. For each n, we repeated our procedure and redetermined the hierarchical structure of the perturbed networks. As shown in Figure 2B, with the increase numbers of perturbations the hierarchy scores of the perturbed networks decrease asymptotically, indicating that the HS is an effective measurement for quantifying the degree of hierarchy of directed networks.

\section{Hierarchical scores of several directed networks}

We next apply the HSM algorithm to calculate the degree of hierarchy of eight different directed networks, including five biological networks (yeast regulatory network, human regulatory network, yeast phosphorylation network, human phosphorylation network and worm neural network), one ecological network (food web), one social network (political blogs), and one computer network (P2P file sharing network) (see 'Materials and methods' for information about these networks). We can evaluate the performance of the HSM algorithm, since we have an intuitive sense of the degree of hierarchy of these non-molecular networks.

In Table 1, we summarize the topological properties of these eight networks being sorted in the increasing order of CHSs. The political blog network contains hyperlinks between weblogs on US politics being recorded in 2005 [3]. The weblogs refer to each other by hyperlinks largely 
Table 1 Hierarchical scores of eight directed networks

\begin{tabular}{|c|c|c|c|c|c|c|c|c|c|c|c|}
\hline Network & Nodes (n) & Edges (n) & Levels (n) & 1-DR & KHS & GRC & HS & $\mathrm{CHS}$ & PHS & Significance $^{a}$ & Reference \\
\hline Worm neural & 297 & 2,359 & $L=4$ & 0.184 & 0.186 & 0.133 & 2.805 & 2.364 & 2.707 & $1.60(30.72)$ & Watts et al. [45] \\
\hline Political blogs & 1,224 & 19,087 & $L=3$ & 0.487 & 0.514 & 0.130 & 3.044 & 3.177 & 2.972 & $2.23(251.38)$ & Adamic et al. [3] \\
\hline Yeast TF & 149 & 580 & $L=4$ & 0.559 & 0.611 & 0.381 & 4.675 & 3.869 & 4.330 & $1.97(22.32)$ & Harbison et al. [10] \\
\hline Human TF & 112 & 513 & $\mathrm{~L}=4$ & 0.631 & 0.718 & 0.336 & 7.087 & 5.608 & 5.848 & $3.15(53.30)$ & Gerstein et al. [23] \\
\hline P2P file sharing & 6,301 & 20,777 & $\mathrm{~L}=4$ & 0.486 & 0.772 & 0.628 & 4.348 & 5.878 & 2.401 & $1.71(74.06)$ & Ripeanu et al. [58] \\
\hline Foodweb & 63 & 612 & $L=3$ & 0.259 & 0.261 & 0.582 & 5.788 & 6.407 & 5.788 & $4.26(190.38)$ & Ulanowicz et al. [46] \\
\hline Human kinase & 373 & 2,171 & $L=4$ & 0.492 & 0.798 & 0.020 & 14.087 & 13.396 & 12.874 & $7.16(275.89)$ & Newman et al. [38] \\
\hline Yeast kinase & 94 & 200 & $L=4$ & 0.645 & 0.775 & 0.447 & 17.455 & 13.982 & 11.777 & $4.81(41.73)$ & Ptacek et al. [11] \\
\hline
\end{tabular}

${ }^{a}$ Significance is calculated by comparing a network with 1,000 Erdos-Renyi random networks. The first number is the ratio of its HS to the average HS of the random networks. The second number in the parenthesis is the t-statistics of the HS. All networks listed in the table are significant $(P<2 \mathrm{e}-16)$.

1-DR: 1-dyadic reciprocity; CHS: corrected hierarchy score; GRC: global reaching centrality; HS: hierarchy score; KHS: Krackhardt hierarchy score; PHS: probabilistic hierarchy score.

in a non-hierarchical manner, and consistently, we observe a relatively low hierarchical structure of it $(\mathrm{CHS}=3.2)$. In contrast, the food web network typically is known to have a pyramidal structure: the number of predators at each level decreases significantly, so that a single top predator is supported by a much larger number of preys. Indeed, the food web network is more hierarchical, with a CHS of 6.4. In addition, the worm neural network is the least hierarchical one among these networks, consistent to our knowledge that neurons are not hierarchically but mutually connected with one another [28].

Our results reveal several interesting findings. First, in both human and yeast, the phosphorylome is more hierarchical than the regulome (Table 1 and Figure 3), although all of these networks show significant hierarchical structures compared to a random network $(P<2 \mathrm{e}-16$, see method for significance estimation). This is seen with the corrected hierarchy scores (CHSs) for yeast regulome and human regulome of 3.9 and 5.6, respectively, in contrast to the CHSs for yeast phosphorylome and human phosphorylome of 13.4 and 14.0, respectively. Surprisingly, the phosphorylomes are even more hierarchical than the food web network. Strikingly, all previous hierarchical network studies have been focused on regulomes and overlooked the phosphorylomes $[17,20,21]$. Our findings suggest that more investigation into the hierarchical nature of phosphorylome is warranted. Second, the degrees of hierarchy for both regulome and phosphorylome are highly consistent between yeast and human, two evolutionarily distant species.

\section{Comparison with other hierarchy construction algorithms}

To compare the HSM algorithm with other methods, we apply it to the yeast regulome which contains 580 regulatory interactions among 145 transcription factors. With the same dataset, $\mathrm{Yu}$ et al. have applied a BFS method to construct a four-level hierarchical network [20]; Jothi et al. have applied a vertex sort (VS) approach to obtain a hierarchical network with seven levels, and further merged them into three levels [21]. We execute the HSM algorithm and obtain hierarchical networks with $3,4, \ldots, 8$ levels. According to the CHSs, the hierarchical network with four levels is the most appropriate one.

We compare the CHSs of the hierarchical networked inferred by different methods (Figure 4A). As HSM is designed to maximize the hierarchical score it gives rise to networks with significantly higher $\mathrm{CHSs}$ than those by BFS and VS methods (Figure 4A). The hierarchical networks inferred by the other two methods have much

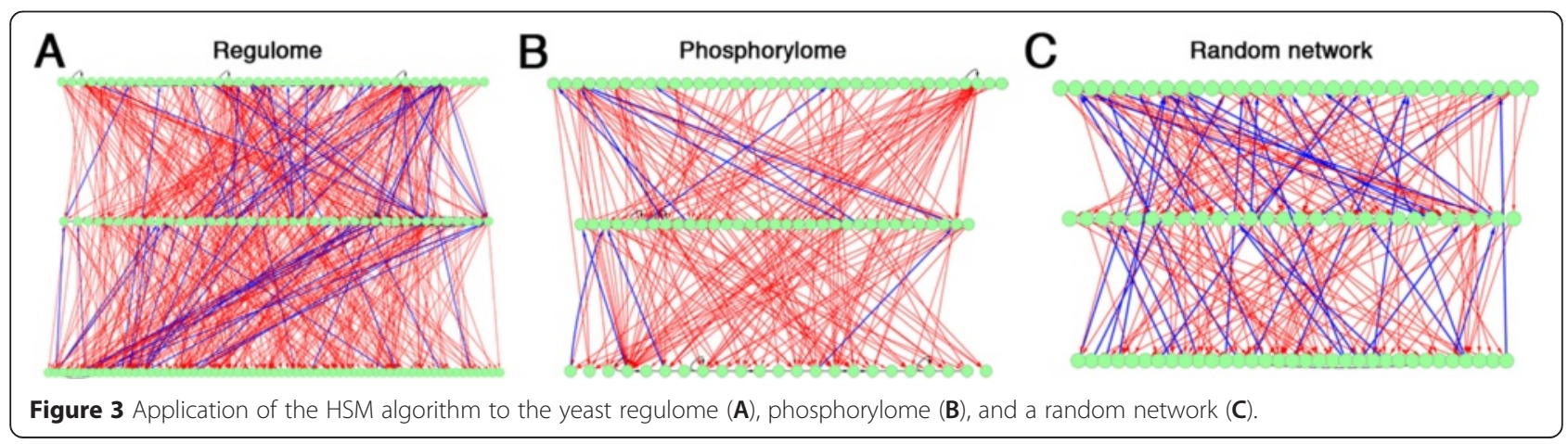




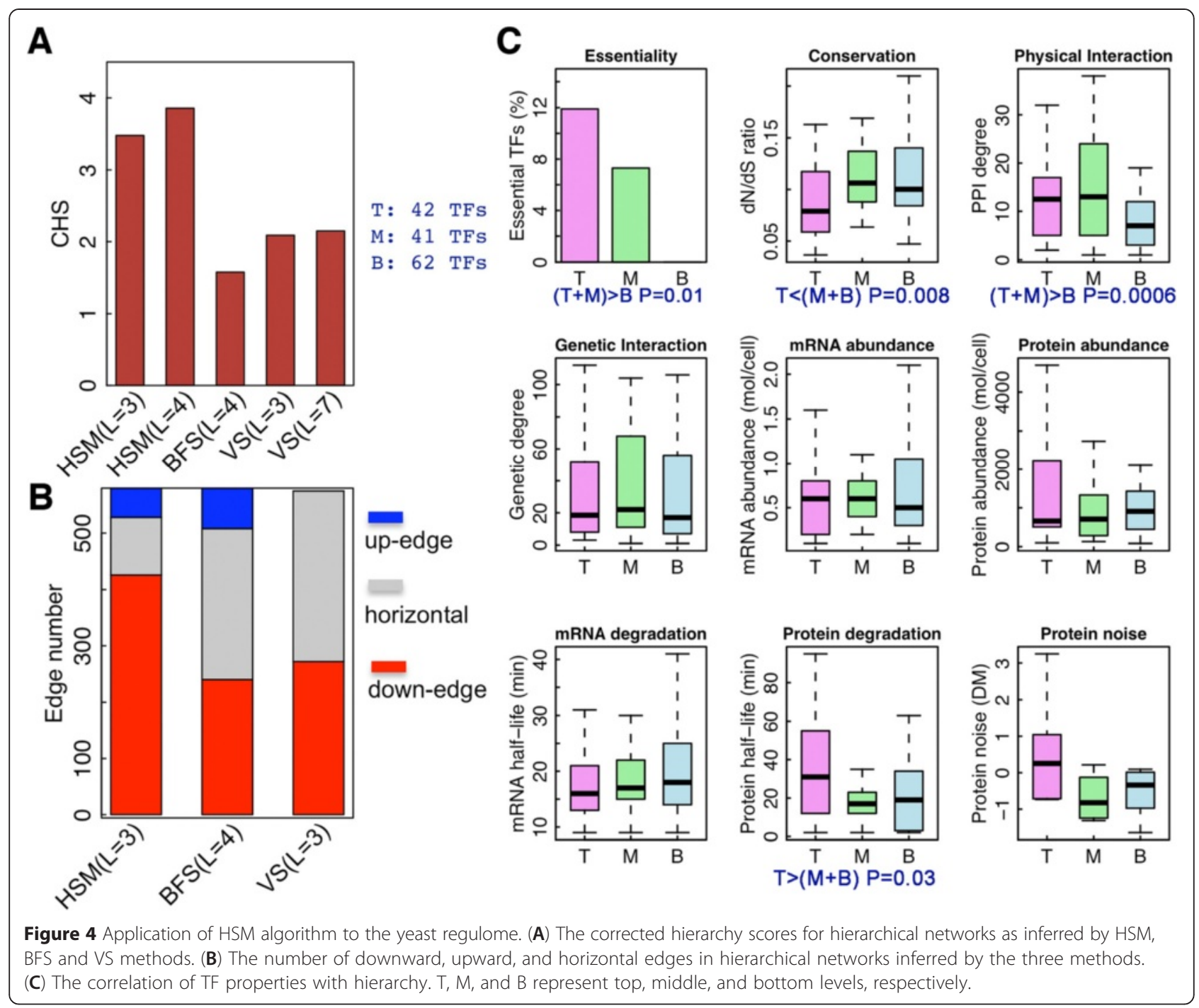

lower CHSs than the optimum score. Moreover, the hierarchical network inferred by the HSM algorithm shows the highest fraction of downward interactions with $>70 \%$ of interactions pointing from higher to lower level TFs. This is in contrast to BFS and VS where $<50 \%$ of interactions are downward. Although there are no upward interactions in the hierarchical network derived from the VS algorithm $(\mathrm{L}=3)$, it has more horizontal interactions than the HSM algorithm (Figure 4B). A similar fraction of horizontal edges are observed in the seven-level hierarchical network inferred by the VS algorithm.

We next examine the properties of TFs in relation to the hierarchy inferred by the HSM algorithm. As shown in Figure 4A, the hierarchical network for the yeast regulome with four levels $(\mathrm{L}=4)$ achieves the highest $\mathrm{CHS}$, but the CHS for the network with three levels $(L=3)$ is just slightly lower. In order to simplify the downstream analysis and to facilitate the comparison with previous studies we focus our analysis on the one with three TF levels, with 42, 41, and 62 TFs at the top, middle, and bottom levels, respectively (Additional file 1: Table S1). First, we compare the percentage of essential TFs in the three levels. Our results indicate that higher level TFs are more likely to be essential: five out of 42 top level TFs (12\%) and three out of 41 middle level TFs (8\%) are essential. In contrast, none of the 62 bottom level TFs is essential $(P=0.01$, Fisher's exact test). In line with this, the TFs at the higher levels are more conserved during the evolution with the top level TFs tend to having a lower $\mathrm{dN} / \mathrm{dS}$ ratio (calculated based on $S$. cerevisae versus $S$. pombe comparison) than the middle and bottom level TFs $(P=0.008$, Wilcoxon rank sum test). These results are consistent with those previously reported in Jothi et al. [21]. Second, we examine the degrees of the TFs at different levels in the physical interaction and 
genetic interaction networks. We find that TFs in higher levels $(T+M)$ have significantly more physical interactions $(P=0.0006$, Wilcoxon rank sum test) than those in the bottom level, consistent with our observations in the human regulome [17]. The average numbers of partners for TFs in different layers rank in the order of $\mathrm{M}>\mathrm{T}>\mathrm{B}$. A similar trend $(M>T>B)$ is observed for genetic interactions, but it does not pass the significance threshold ( $P>0.05$ when TFs in $\mathrm{T}+\mathrm{M}$ layers are compared to those in $B$ layer in terms of number of genetic interactions). Third, we compare the TFs at different levels on their dynamic properties, including their abundance and stability at both the mRNA and protein level, and their protein expression noise. The results indicate that the top-level TFs are more stable than middle- and bottomlevel TFs $(P=0.03$, Wilcoxon rank sum test) (Figure $4 \mathrm{C}$ ). Overall, our results highlight the critical roles played by the top-level TFs, as also reported by Jothi et al. using the VS algorithm [21]. These master regulators are highly conserved during evolution with a higher essentiality rate.

\section{Features of kinases at different levels}

Our results suggest that the organization of phosphorylomes is more hierarchical than the regulomes. We infer the hierarchical structure of the yeast phosphorylome by using the HSM algorithm. This network is mainly based on protein chip experiments and contains 200 phosphorylation interactions among 94 different kinases [11,29]. Again, for easy comparison we specify the number of hierarchical levels $\mathrm{L}=3$, which results in 38 top-level, 33 middle-level, and 23 bottom-level kinases (Additional file 2: Table S2).

We examine the cellular localization according to the Saccharomyces genome database, which are manually annotated based on previous literatures. Of the $94 \mathrm{ki}-$ nases 35 localize only to the cytoplasm, eight only to the nucleus, and 12 to both (the remaining 39 kinases are in other locations or localization unknown). Interestingly, the kinases in the middle level are more likely to localize in both nucleus and cytoplasm compared to the top and bottom level kinases $(P=0.02$, Fisher's exact test, Figure $5 \mathrm{~A})$. Gene ontology analysis suggests that the top level is
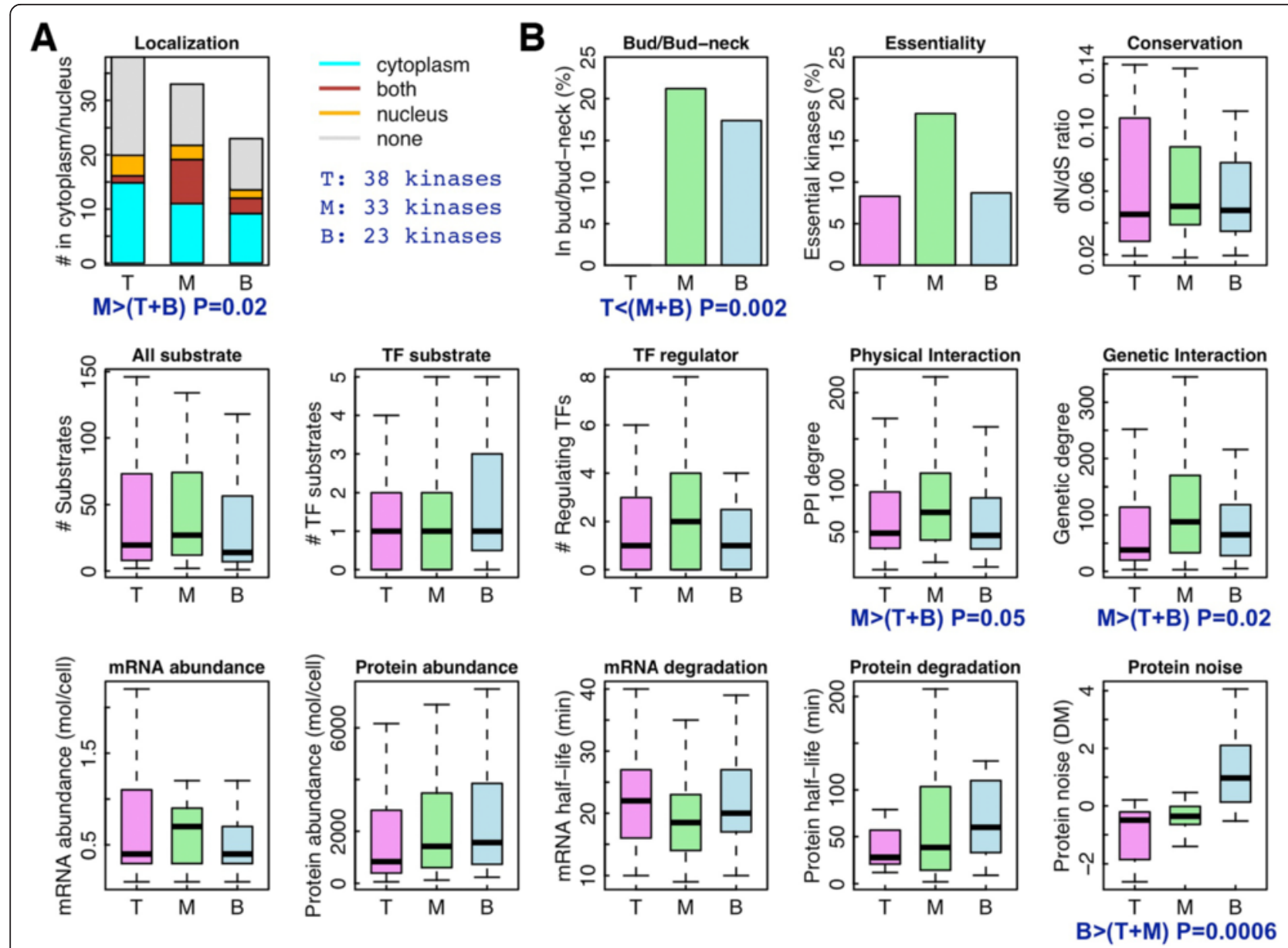

Figure 5 Application of the HSM algorithm to the yeast phosphorylome. (A) The localization of kinases at different levels in the cytoplasm and nucleus. (B) The correlation of kinase properties with hierarchy. T, M, and B represent top, middle, and bottom levels, respectively. 
enriched in trans-membrane proteins and stress-response proteins implying that the top-level kinases tend to be located in the cell membrane and respond to extracellular signals (Additional file 3: Table S3). In contrast, the middle level is enriched in cell cycle related kinases.

We also relate the hierarchical structure of the yeast phosphorylome with a number of kinase properties (Figure 5B). Our findings are summarized as follows: (1) The bud/bud-neck located proteins are highly enriched in kinases of the middle and bottom levels with respect to the top level $(P=0.002$, Fisher's exact test). Strikingly, none of the 38 top-level kinases is a bud/bud-neck protein. This may suggest that during yeast budding the top-level kinases function mainly in the mother cells rather than enter the bud/bud-neck to perform as direct effectors. (2) The middle-level kinases show higher essentiality rate $(18 \%)$ than the top-level (8\%) and the bottom-level (8\%) kinases. (3) Kinases in the middle level have significantly more physical $(P=0.05$, Wilcoxon rank sum test) and genetic $(P=0.02$, Wilcoxon rank sum test) interaction partners. (4) The bottom-level kinases are significantly noisier in their protein abundance than kinases in the higher levels $(P=0.006$, Wilcoxon rank sum test).

\section{Collaboration of kinases in different levels}

We next explore how kinases in the top, middle, and bottom levels collaborate with one another, in terms of both inter-level (TM, MB, TB) and intra-level (TT, MM, $\mathrm{BB})$ relationships. First, we examine the physical and genetic interactions between kinases at different levels. Our results show that physical interactions are significantly enriched in TB (between top-level and bottomlevel kinases) and $\mathrm{MB}$ (between middle-level and bottom-level kinases), but depleted in the intra-level relationships (TT, MM, and $\mathrm{BB}$ ). The genetic interactions are significantly enriched in $\mathrm{MB}$, and depleted in TT and TB relationships (Figure 6A). This suggests that inter- level interactions between kinases, particularly between middle- and bottom-level kinases, are dominant over those intra-level interactions.

Second, we investigate kinase cooperativity. We define two kinases as being cooperative if they share a significantly large number of physical partners, genetic partners, or phosphorylation substrates (Figure 6B). We find that physical cooperation between kinases is enriched in $\mathrm{TB}$, while genetic cooperation is enriched in $\mathrm{MB}$ relationships. Interestingly, cooperation is highly depleted between bottom level kinases suggesting that, as downstream effectors, they tend to phosphorylate different subsets of proteins to take specific effects. Finally, we further divide genetic interactions into positive and negative ones, and examine their enrichment or depletion between kinases. Positive and negative genetic interactions involve a pair of genes with mutations or deletions in which each alone causes a minimal phenotype, but when combined in the same cell results in a less severe (positive) or a more severe (negative) fitness defect than expected under a given condition [30]. As shown in Figure $6 \mathrm{C}$, both positive and negative genetic interactions are significantly enriched in $\mathrm{MB}$ relationships.

\section{Substrate of kinases at different levels}

The network contains 200 inter-kinase phosphorylation interactions (one kinase phosphorylating another) and six auto-phosphorylation interactions (CKA2, TPK2, RAD53, PRP1, CDC7, and CDC15). Indeed, the autophosphorylation is over-represented in the network $(P=0.02$, see 'Materials and methods' for details). There are two feedback loops (TPK2 and TPK3, ELM2 and GIN4) involving two kinases in the network in which the two kinases phosphorylate each other. The feed-forward loop (FFL) network motif is highly enriched in the yeast phosphorylome. We investigate the FFL motifs in the context of hierarchy. In a FFL with three nodes, one kinase phosphorylates another kinase and both target a third
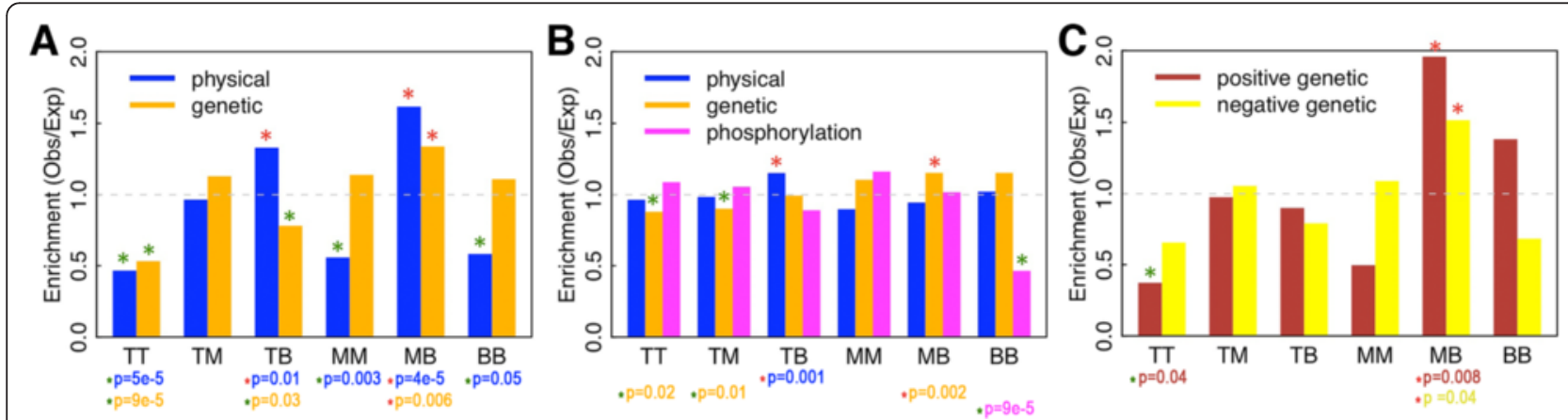

Figure 6 Collaboration of kinases at different hierarchy levels. (A) The enrichment of physical and genetic interactions of kinases within a level ( $T T, M M$, and $B B$ ) and between two levels (TM, TB, and MB). (B) The enrichment of kinase pairs with significantly overlapping physical or genetic interaction partners or phosphorylation substrates. (C) The enrichment of positive and negative genetic interactions of kinases. Enrichment and depletion of interactions $(P<0.05)$ are marked as red and green '*', respectively. 
protein as substrate, which can be either a kinase or nonkinase. We enumerate all of three-node FFL motifs in the yeast phosphorylation data (including non-kinase substrates) and map the two kinases in these motifs to the hierarchical network. Each of the two kinases in a FFL motif can be from one of the three hierarchical levels ( $T$, $\mathrm{M}$, and $\mathrm{B}$ ), which results in nine combinations (TT, TM, $\mathrm{TB}, \mathrm{MT}, \mathrm{MM}, \mathrm{MB}, \mathrm{BT}, \mathrm{BM}$, and $\mathrm{BB})$. We count the number of FFL motifs for all the nine types and our results show that $>90 \%$ FFL motifs involve downward interactions between kinases in the hierarchical networks (Figure 7A, red bars). The TM type FFL motif, in which a top-level kinase phosphorylates a middle-level kinase and both kinases share a target substrate, is significantly enriched.

We also examine and compare the functions of the substrate targets of kinases at different levels. The 38 top-level kinases target a total of 1,095 substrates; the 33 middle-level kinases target 998 substrates; and the 23 bottom-level kinases target 612 substrates. The substrate targets of the three levels highly overlap as shown in Figure 7B. After filtering out the shared substrate targets, we identify 294 top-level, 228 middle-level, and 159 bottom-level specific substrate targets. Gene ontology analysis indicates that the top-level specific substrates are enriched in gene categories involving in 'protein kinase activity', 'phosphorylation', and 'phosphate metabolic process', and so on (Additional file 4: Table S4). In other words, the top-level kinases are involved in the regulation of other phosphorylationrelated proteins. In contrast, the middle- and bottomlevel specific substrate targets are enriched in structural proteins, for example, gene categories involving in 'microtubule cytoskeleton', 'structural molecule activity', and 'macromolecular complex subunit organization'.

\section{Discussion}

\section{Global optimization versus local optimization}

To determine the hierarchical structure of a directed network, the leaf removal and the BFS methods apply a local optimization strategy. The leaf removal algorithm employs a bottom-up iterative procedure. It assigns all the leaf nodes (nodes with zero out-degree) to the bottom level, and then iteratively removes all the leaf nodes and the edges associated with them from the network to determine the next higher level [25]. The BFS method also starts by assigning the leaf nodes to the bottom level, and then performs a BFS to define the level of a non-bottom node as its shortest distance from a bottom one [20]. In contrast, the hierarchical score maximization (HSM) algorithm presented here works to globally optimize the hierarchy of a directed network. It defines a hierarchy score (HS) to quantify the degree of hierarchy in a network. The hierarchical score captures the global hierarchical property of a network. To infer the hierarchy, HSM optimizes the hierarchical structure so that the maximum HS is achieved. Thus, the hierarchy inferred by HSM represents the globally optimized structure. The VS algorithm identifies strongly connected components and collapses them to convert the network into a directed acyclic graph, and applies the leaf removal algorithm on the graph and on its transpose. Results are then combined to infer a global solution of hierarchical levels [21]. This method avoids any upward edges but the resulting networks have smaller hierarchy scores compared to those from the HSM algorithm.

Compared with the previous methods, the HSM algorithm takes into account the potential hierarchical ambiguity underlying a network. It provides a probabilistic representation of the hierarchy for a network that can more precisely reflect the underlying hierarchical
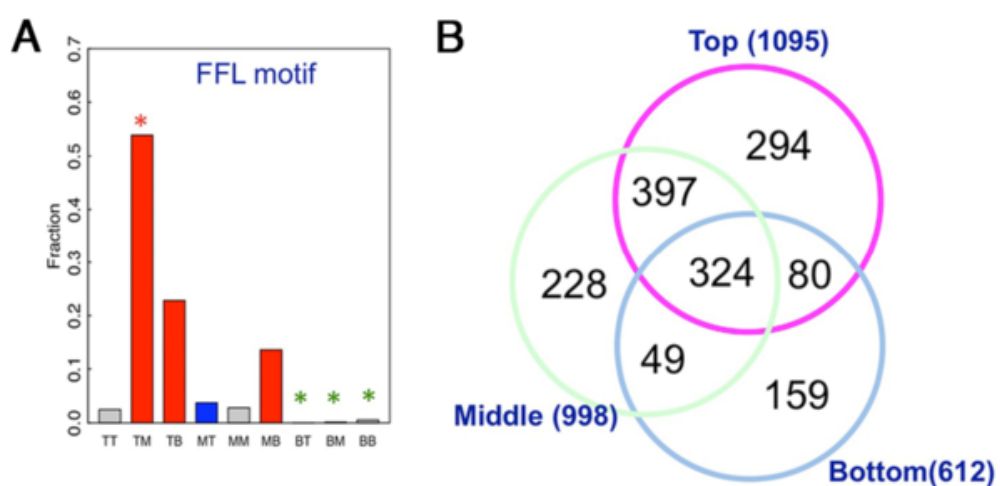

Figure 7 Properties of the inferred hierarchical structure for the yeast phosphorylome generated by HSM algorithm. (A) The distribution of feed-forward loop (FFL) motifs in the hierarchical network. In a FFL motif, a kinase X phosphorylates another kinase $\mathrm{Y}$ and both target a common substrate $Z$. Depending on the location of $X$ and $Y$ in the hierarchical structure, the $X->Y$ interaction can be categorized into nine combinations. Downward interactions (TM, TB, and MB), upward interactions (MT, BT, and BM), and horizontal interactions (TT, MM, and BB) are shown with red, blue, and gray bars, respectively. (B) The Venn diagram of substrates of kinases at different levels. 
structure. For all the nodes, we know the certainty of them being assigned to a hierarchical level, which is informative and is useful for us to interpret their roles in the hierarchical network. A global optimization method has been proposed in [27], which applied a simulated annealing algorithm to minimize the number of 'backward' links going from lower to higher hierarchical levels. In contrast, we define a hierarchical score that quantifies the degree of hierarchy and infer the hierarchical structure of a network by the scores magnitude.

Moreover, the HSM algorithm's corrected hierarchical score (CHS) is comparable between different networks. As shown in Table 1, this enables comparisons in the degree of hierarchy between different biological networks such as social networks, file sharing networks, ecological networks, and neural networks. Practically, this allows for the exploration of the common rules shared by different networks and reveals the differences between them [31]. For example, we find that the protein phosphorylation interactions mediated by kinases are much more hierarchical than the transcriptional regulatory interactions mediated by TFs.

\section{Hierarchy versus asymmetry for directed networks}

Dyadic reciprocity and Krackhardt hierarchy score are often used to quantify the extent of asymmetry in directed networks [32]. The former is defined as the proportion of node pairs that are reachable from either direction, while the latter is the fraction of node pairs that are reachable from only one direction. We note that Krackhardt hierarchy score, though termed a 'hierarchy' score, is distinct from the hierarchy score (HS) described here. The asymmetry measured by reciprocity or Krackhardt score quantifies the degree to which two nodes are 'mutually reachable' in a directed network. A related metric called global reaching centrality (GRC) was defined to measure hierarchy as heterogeneous distribution of the local reaching centrality (the proportion of all nodes that can be reached from a node) of all nodes in a directed network [33]. These metrics do not imply any information on orientation. In contrast, by hierarchy here we mean a top-to-bottom orientation for nodes at different levels.

Why do we need to introduce the 'orientation/hierarchy' attribute for a directed network? Because in many networks the nodes are by nature associated with certain 'spatial' or 'temporal' attributes. For example, the protein nodes in a biology network may localize in different cellular components, for example, the membrane, cytoplasm, or nucleus; meanwhile, external signals are often transduced following a specific direction from membrane to nucleus. This confers a global 'hierarchy' attribute to the network that cannot be captured by 'asymmetry' attributes (for example, reciprocity and
Krackhardt score). On the other hand, since the 'hierarchy' originates from certain attributes of nodes, we would expect to observe the correlation of hierarchy with node features. In other words, the inferred hierarchical structure should recapitulate the attribute difference of nodes at different levels. For instance, as shown in Figure 4, we find that the higher-level TFs in the yeast regulome are more likely to be essential and more conserved.

The hierarchy score is also different than the threedimensional 'morphospace' proposed recently by CorominasMurtra, which defines three hierarchical features: treeness, feed-forwardness, and orderability [34]. To define them, nodes with zero in-degrees and out-degrees are regarded as the source and the sink of a network, respectively, and then the paths between them are characterized.

\section{Temporal versus spatial organization of hierarchy}

We observe interesting results when we apply the HSM algorithm to the yeast regulome and phosphorylome. In the yeast regulome, we find that higher-level TFs are more likely to be essential and are more conserved during evolution. Particularly, none of the 62 bottom-level TFs are essential, compared to an average essentiality rate of $19 \%$ in yeast. In the yeast phosphorylome, however, this is not the case and instead we observe significant differences in cellular localization for kinases at different levels. For instance, $21 \%$ of the middle-level and $18 \%$ of the bottom-level kinases are detectable in bud/bud-neck, whereas in the 38 top-level kinases, none are identified in bud/bud-neck.

Biologically, the hierarchy of regulatory networks (regulome and phosphorylome) may arise from the temporal and/or spatial organization of regulators. In response to stimulation or in a biological process (for example, cell cycle regulation), early-activated regulators (for example, TFs or kinases) regulate the expression/activation of later regulators, which in turn regulate even later ones, forming a hierarchical structure. Similarly, the cellular localization of regulators can also contribute to the hierarchical organization of a regulatory network. For example, during signal transduction the extracellular signal is typically transferred from a membrane-localized kinase to a cytoplasmic kinase and onward to a nuclear kinase [35]. Since in general TFs function in the nucleus by regulating gene expression, their hierarchy is mainly organized via temporal activation of TFs. However, in phosphorylomes the hierarchical organization of kinases can be determined by both temporal regulation and spatial localization. The differential correlation pattern of protein features with hierarchy between regulomes and phosphorylomes may reflect such a difference.

One caveat of this study involves the quality of data used for constructing the phosphorylomes. The yeast 
phosphorylome is constructed based on two sources: kinase-substrate interactions identified from protein chip experiments by Ptacek et al. [11] and phosphorylation site data collected by Freschi et al. from several largescale studies [36]. The Patcek data are derived from in vitro measurements of kinase-substrate interactions, some of which may not necessarily occur in vivo. The Freschi data derived from a set of experimentally identified phosphorylation sites, for which the associated protein kinases were computationally predicted by matching with position weight matrices of yeast kinases. Thus, we would expect a high false positive rate in the yeast phosphorylome, and, similarly, this is also the case for human phosphorylome. More confident kinase-substrate interactions might be obtained by selecting those in which kinase and substrate are present in the same cellular compartment or in the same functional categories. However, this filtering procedure may also increase the number of false negatives since the protein localization data and function annotation may be incomplete or inaccurate. In addition, only a subset of kinase-substrate interactions have been identified and included in the phosphorylomes. Therefore, a more detailed analysis should be performed to validate findings in this analysis when more complete and accurate phosphorylation data become available in the future.

\section{Conclusions}

In summary, the HSM algorithm provides a useful tool to investigate the hierarchy of directed networks. It can be used independently or in conjunction with other hierarchy inference methods. With more and various regulatory interaction data being generated, we expect a wide application of these methods in biological network studies.

\section{Materials and methods}

\section{Construction of network hierarchy}

A hierarchical network is a directed network for which all nodes are assigned to a unique hierarchical level from 1 to $\mathrm{L}$, where $\mathrm{L}$ is the total number of levels $(\mathrm{L} \geq 2)$. Generally, a hierarchical network contains three types of edges according to their directionality: a downward edge (pointing from a higher level node to a lower level node), an upward edge (pointing from a higher level node to a lower level node), and a horizontal edge (pointing from a node to another node in the same level). To infer the hierarchy of a directed network, we developed a hierarchical score maximization algorithm described as follows.

First, given a directed network with assigned hierarchical structure, we define a metric called hierarchy score as:

$H S=\frac{N_{d}+N_{h}}{N_{u}+N_{h}}$, where $\mathrm{N}_{\mathrm{d}}, \mathrm{N}_{\mathrm{u}}$, and $\mathrm{N}_{\mathrm{h}}$ are the number of downward edges, upward edges, and horizontal edges, respectively. The metric essentially measures the ratio of $\mathrm{N}_{\mathrm{d}}$ to $\mathrm{N}_{\mathrm{u}}$ balanced by $\mathrm{N}_{\mathrm{h}}$. It takes a value from 0 to $+\infty$, with a higher HS indicating more downward edges relative to upward edges in a network. Specifically, when $\mathrm{N}_{\mathrm{u}}=\mathrm{N}_{\mathrm{h}}=0$, the network will have a HS of $+\infty$.

Second, for a directed network we employ a simulated annealing procedure [37] to infer its hierarchical structure by arranging nodes into $\mathrm{L}$ levels ( $\mathrm{L}$ is a pre-defined parameter). This procedure is as follows:

(1) We initiate from randomly assigning each node to a level, calculate the corresponding HS score $\mathrm{hs}_{0}$, and setting the initial energy as $\mathrm{E}_{0}=-\mathrm{hs}_{0}$.

(2)We adjust the hierarchy iteratively to optimize the hierarchical structure. Specifically, at iteration i, we randomly select a node, adjust the hierarchy by randomly placing it into another level and recalculate the hierarchical score and energy $\left(\mathrm{hs}_{\mathrm{i}}=-\mathrm{E}_{\mathrm{i}}\right)$ of the resulting new hierarchy. We compute the energy change $\Delta \mathrm{E}=\mathrm{E}_{\mathrm{i}}-\mathrm{E}_{\mathrm{i}-1}$; if $\Delta \mathrm{E}<0$, we accept the hierarchy adjustment; otherwise we accept the adjustment with a probability $P=\exp (-\Delta \mathrm{E} / \mathrm{CT})$, where $\mathrm{C}$ is a constant and $\mathrm{T}$ is temperature that are used to tune the probability $P$.

(3) We repeat this procedure $\mathrm{p}$ times until $\mathrm{E}$ is minimized (that is, HS is maximized). In practice, we gradually lower the temperature $\mathrm{T}$ at each step to adjust the sensitivity of annealing. This procedure results in an optimized hierarchical network with maximized HS score.

Third, we perform the above-described simulated annealing algorithm $\mathrm{k}=1,000$ times to obtain 1,000 inferred hierarchical networks. We do this because in many cases the optimum hierarchy is not unique. For example, some nodes are topologically identical in a directed network, and changing their level assignment coordinately will not change the overall hierarchical score. Based on these 1,000 inferred hierarchical networks, we calculate the probability that each node is assigned to each level, which results in a probability matrix for each node as seen in Figure 1C. This matrix can be regarded as a probabilistic hierarchical network, which is more informative and more precisely describes the hierarchical structure of a directed network than methods that omit this procedure.

Fourth, we provide a most likely hierarchical network based on the probabilistic hierarchical matrix. Specifically, we assign each node to the level for which the prior step assigns it the highest probability. It should be noted that the confidence of the assignment might vary from node to node, depending on the value of the maximum probability. Typically, however, most of the nodes have high certainty in terms of the level assignment (for example, the probability in the assigned level is $>60 \%$ ). 
To determine an appropriate $\mathrm{p}$ (the number of steps in each simulated annealing procedure) and $\mathrm{k}$ (the number of each simulated annealing runs), we plot the hierarchy score against $\mathrm{p}$ and $\mathrm{k}$, respectively. For a network with more nodes and edges, a larger $\mathrm{p}$ should be used as can be determined based on the HS vs. p plot. When a suitable $\mathrm{p}$ is used, the resulting $\mathrm{HS}$ should be stable against $\mathrm{k}$ when $\mathrm{k}$ is $>100$.

In practice, the HSM method can be used conjunction with other hierarchy inference methods. For example, one may start from the hierarchical structure inferred by the VS algorithm, and use the simulated annealing procedure method to further optimize the hierarchy score. Namely, instead of randomly selecting nodes during the simulated annealing optimization, we can focus on adjusting the levels of ambiguous nodes from VS output to improve the efficiency. Such a strategy will combine the advantages of the two hierarchy inference approaches.

\section{Determination of the number of hierarchical levels}

The HSM algorithm requires a pre-defined L, the number of hierarchical levels. L can be determined based on the prior knowledge about the directed network of interest. If no prior knowledge is available, we can specify different $L$ values (e.g. $L=2,3, \ldots, 8$ ) and choose a proper $\mathrm{L}$ by comparing the resulting hierarchical networks. However, the HS score is not directly comparable for hierarchical networks with different number of levels, because networks with larger L tend to have higher HSs. We thereby define a corrected hierarchical score (CHS) as the following:

$$
C H S=\frac{O\left(N_{d}\right) / E\left(N_{d}\right)+O\left(N_{h}\right) / E\left(N_{h}\right)}{O\left(N_{u}\right) / E\left(N_{u}\right)+O\left(N_{h}\right) / E\left(N_{h}\right)},
$$

where $\mathrm{O}\left(\mathrm{N}_{\mathrm{d}}\right), \mathrm{O}\left(\mathrm{N}_{\mathrm{u}}\right)$, and $\mathrm{O}\left(\mathrm{N}_{\mathrm{h}}\right)$ are the observed number of downward, upward, and horizontal edges, respectively; $\mathrm{E}\left(\mathrm{N}_{\mathrm{d}}\right), \mathrm{E}\left(\mathrm{N}_{\mathrm{u}}\right)$, and $\mathrm{E}\left(\mathrm{N}_{\mathrm{h}}\right)$ are the expected number of downward, upward, and horizontal edges, respectively. $\mathrm{E}\left(\mathrm{N}_{\mathrm{d}}\right), \mathrm{E}\left(\mathrm{N}_{\mathrm{u}}\right)$, and $\mathrm{E}\left(\mathrm{N}_{\mathrm{h}}\right)$ are calculated as:

$$
\begin{aligned}
& E\left(N_{d}\right)=\sum_{i>j} S_{i} S_{j} ; \\
& E\left(N_{u}\right)=\sum_{i<j} S_{i} S_{j} ; \\
& E\left(N_{h}\right)=\sum_{i=j} S_{i} S_{j},
\end{aligned}
$$

where $\mathrm{S}_{\mathrm{i}}$ and $\mathrm{S}_{\mathrm{j}}$ are the number of nodes in level $i$ and level $j$, respectively. The CHS is directly comparable between hierarchical networks with different $\mathrm{L}$ values, and can also be used to compare the degree of hierarchy between different directed networks. The CHS takes a value from 1 for random network without a hierarchical structure to $\infty$ for a network with a perfect hierarchy (for example, a tree as in Figure 2).

To determine the number of hierarchical level $\mathrm{L}$ for a network, one can employ the HSM algorithm across a range of $\mathrm{L}$ values and choose the L for which the HSM algorithm yields the highest CHS. In some cases, the CHS will keep increasing with the increase of $\mathrm{L}$, because there is more freedom to optimize the hierarchy with larger $\mathrm{L}$ values. In this situation, one can plot the CHS against $\mathrm{L}$ values, and choose the $\mathrm{L}$ at which no significant $\mathrm{CHS}$ improvement is achieved. In addition, other information is also important to determine the $\mathrm{L}$ for a directed network. For example, it is reasonable to require $\mathrm{L}$ to be no larger than the diameter of the network, namely, the greatest distance between any pair of connected nodes.

\section{Calculation of probabilistic hierarchical score}

To more accurately measure the hierarchical structure of a probabilistic hierarchical network, we define a new metric called the probabilistic hierarchical score (PHS). For an edge $i \rightarrow j$ in a network with L levels, the probability of this edge being downward is $\sum_{L_{i}>L_{j}} P\left(L_{i}, i\right) P\left(L_{j}, j\right)$, where $P\left(L_{i}, i\right)$ and $P\left(L_{j}, j\right)$ are the probability of the node $i$ and $j$ in level $L_{i}$ and $L_{j}$, respectively. Similarly, the probability of $i \rightarrow j$ to be upward is $\sum_{L_{i}<L_{j}} P\left(L_{i}, i\right) P\left(L_{j}, j\right)$; and the probability of $i \rightarrow j$ to be horizontal is $\sum_{L_{i}<L_{j}} P\left(L_{i}, i\right) P\left(L_{j}, j\right)$. Thus after taking into account all edges in the network, we define PHS as the following:

$P H S=\frac{\sum_{(i \rightarrow j) \in\{e\}} \sum_{L_{i}>L_{j}} P\left(L_{i}, i\right) P\left(L_{j}, j\right)+\sum_{(i \rightarrow j) \in\{e\}} \sum_{L_{i}=L_{j}} P\left(L_{i}, i\right) P\left(L_{j}, j\right)}{\sum_{(i \rightarrow j) \in\{e\}} \sum_{L_{i}<L_{j}} P\left(L_{i}, i\right) P\left(L_{j}, j\right)+\sum_{(i \rightarrow j) \in\{e\}} \sum_{L_{i}=L_{j}} P\left(L_{i}, i\right) P\left(L_{j}, j\right)}$.

The level is indexed in an increasing order from bottom to top. Namely, level $i$ is higher than level $j$ in the hierarchy, if $i>j$.

\section{Estimation of the hierarchy significance for a directed network}

Given a directed network, the HSM algorithm infers its optimum hierarchical structure by maximizing the HS score. Although the resulting HS score can measure the degree of hierarchy of a network, it does not tell us whether a directed network has a significantly hierarchical structure. To address this issue, we compare a directed network with random networks to evaluate its hierarchical significance. Here we use the Erdos-Renyi random graph model as the null model. In a network, each pair of nodes has an equal chance to be connected by an edge [38]. We generate 1,000 Erdos-Renyi random networks with the same number of nodes and edges, and calculate their HSs using the HSM algorithm. Given 
a specified number of layers (L), the $P$ value of hierarchy for the network of interest is then computed as the fraction of random networks with a HS (the same L is specified) equal to or greater than the interested network. Alternatively, assuming a Gaussian distribution of the HSs of the random networks, we calculate the Z-score for the interested network: $Z=(\mathrm{HS}-\mu) / \sigma$, where $\mu$ and $\sigma$ are the mean and standard deviation of the HS scores of those random networks with $\mathrm{L}$ levels; the $P$ value is calculated by referring to a standard normal distribution, and for each $\mathrm{L}$ a corresponding $P$ value is calculated $(\mathrm{L}=2,3, \ldots, 8)$. The hierarchy for the whole network is calculated as the minimum P-value adjusted by the Bonferroni multiple-testing correction method. We note that the significance estimation depends on the selection of the null model. To generate the random networks, other null models can be used and certain constraints can be applied as required.

\section{Calculation of dyadic reciprocity and Krackhardt hierarchy score}

Traditionally, 1-dyadic reciprocity and Krackhardt hierarchy score are often used to quantify the extent of asymmetry in directed network [32]. The dyadic reciprocity is defined as the proportion of node pairs in a directed network that are symmetric (that is, reachable from either direction). Krackhardt hierarchy score is the fraction of node pairs in the directed network that are reachable from one direction. These two metrics measure the degree of asymmetry of a directed network, which is different from the hierarchy we introduce in this study. Our hierarchy by nature implies a top-tobottom orientation, whereas the 'asymmetry' is nondirectional. We use the $\mathrm{R}$ package 'sna' to calculate dyadic reciprocity and Krackhardt hierarchy score. The global reaching centrality of networks is calculated using the method introduced by Mones et al. [33].

\section{Directed networks used in this study}

In this study, we examine eight directed networks, including five biological networks, one ecological network (food web network), one social network (political blogs network), and one computer network (P2P file sharing network). The five biological networks are the yeast regulation network, the human regulation network, the yeast phosphorylation network, the human phosphorylation network, and the worm neural network.

The yeast regulome was downloaded from Jothi et al. [21], in which most of the TF-gene interactions were identified by ChIP-chip experiments $[9,10]$, and the remaining were collected based on other biochemical studies [39-42]. The human regulome is constructed based on ChIP-seq data from the ENCODE project [43], based on which the target genes of more than 120 TFs are determined by a probabilistic model [44]. For TFs with multiple ChIP-seq datasets, the target genes represent a union of targets in all of the available datasets. The kinase-substrate interactions in the yeast phosphorylome are collected from protein chip experiment by Ptacek et al. [11] and the phosphorylation site data collected by Freschi et al. from several large-scale studies [36]. The human phosphorylome is available from the PhosphoNetworks database, which is based on experimental determined kinasesubstrate relationships [38]. In our hierarchical study, we include only TF-TF interactions in the two regulomes and kinase-kinase interactions in the two phosphorylomes.

The worm neural network contains the interaction of one neuron to another via synaptic or gap junctions in worm [45]. The food web network is from Ulanowicz et al., which contains the carbon exchange from one species to another occurring during the wet season in the cypress wetlands of south Florida [46]. The Political blogs network contains hyperlinks between weblogs on US politics being recorded in 2005 [3]. The P2P filesharing network is one of a series of Gnutella network created in 2002, in which nodes represent host computers in the Gnutella computer network and edges represent connections between the hosts [47].

\section{Properties of yeast genes and proteins}

The list of yeast essential genes was determined by a yeast gene deletion project and was downloaded from the Saccharomyces genome database (SGD) [48]. The $\mathrm{Ka} / \mathrm{Ks}$ ratios of ortholog genes between S. cerevisiae and S. pombe orthologs were from Wall et al. [49]. The physical and genetic interactions of yeast genes were also downloaded from the SGD database [50,51]. Specifically, to calculate the number of physical interacting partners of yeast kinases the protein-protein interactions between yeast kinases were obtained from Breitkreutz et al. [12]. The mRNA abundance and mRNA half-life data were obtained from previous studies [52,53]. The protein half-life data came from Belle et al. [54]. The protein abundance and protein noise data were available from Newman $e t$ al. [55]. To determine the protein noise, the single cell expression level of a protein was measured in a population of yeast cells and then the ratio of the standard deviation to its mean abundance was calculated. For a protein, the noise is represented as the difference between its noise value and the median over all proteins, named as deviation from median (DM). Budding or budding neck localization of yeast kinases was obtained from Huh et al. [56]. The cellular component associated with yeast kinases was annotated by SGD, which are manually curated based on previous publications.

\section{Enrichment of interactions between different levels}

To examine whether TFs/kinases are more likely to physically/genetically interact within the same level or 
between two levels (Figure 6A), we calculated the enrichment of interactions in all pairs of levels: TT, TM, $\mathrm{TB}, \mathrm{MM}, \mathrm{MB}$, and $\mathrm{BB}$. Using physical interactions between TFs as the example, the significance of enrichment or depletion is calculated as follows. First, given a physical interaction network with $\mathrm{n}$ nodes and e edges, we compute the probability for a pair of randomly selected genes to interact: $\mathrm{p}=\mathrm{e} /[\mathrm{n}(\mathrm{n}-1) / 2]$. Second, we assume that the number of TF-TF interactions (denoted as $i$ ) within a level or between two different levels follows a binomial distribution: $\operatorname{Pr}(x=i)=f(i ; b, p)=C_{b}^{i} p^{i}(1-p)^{b-i}$, where $\mathrm{b}$ is the number of all possible TF-TF pairs. Considering self-interactions, $b=m(m+1) / 2$ for intra-level interactions with $\mathrm{m}$ TFs (that is, TT, $\mathrm{MM}$, or $\mathrm{BB}$ ), and $\mathrm{b}=$ $\mathrm{m}_{1} \mathrm{~m}_{2}$ for interactions between two levels with $\mathrm{m}_{1}$ and $\mathrm{m}_{2}$ TFs, respectively (that is, TM, TB, and MB). Finally, the $P$ values are calculated as $\mathrm{P}(\mathrm{x} \geq i)$ for enrichment (that is, the probability of observing an equal or greater number of interactions) and $\mathrm{P}(\mathrm{x} \leq i)$ for depletion (that is, the probability of observing an equal or smaller number of interactions) of physical interactions between these TFs.

To estimate whether two kinases share a significantly large number of physical partners, genetic partners or substrates (Figure 6B), we examine their degree of overlap and calculate its significance using Fisher's exact test (that is, hyper-geometric test).

\section{Gene ontology analysis}

We used the DAVID Gene Ontology (GO) annotation tool [57] to investigate the functional enrichment of kinases in the three levels of our hierarchical network for phosphorylome (Figure 3B). The whole list of the $94 \mathrm{ki}-$ nases in the network is used as the background for enrichment analysis. A similar analysis is also used to study the functional enrichment of substrates specific to kinases from each of the three levels. In this case, we use the whole yeast gene list as the background. $\mathrm{R}$ code for this analysis is available from http://gersteinlab.org/proj/hinet.

\section{Additional files}

Additional file 1: Table S1. The hierarchical structure of the yeast regulome with three levels: 3 - Top, 2 - Middle, 1 - Bottom.

Additional file 2: Table S2. The hierarchical structure of the yeast phosphorylome with three levels: 3 - Top, 2 - Middle, 1 - Bottom.

Additional file 3: Table S3. $\mathrm{GO}$ analysis results of kinases in the top, middle, and bottom levels.

Additional file 4: Table S4. GO analysis results of specific substrate proteins of kinases in the top, middle, and bottom levels.

\section{Competing interests}

The authors declare that they have no competing interests.

\section{Authors' contributions}

CC conceived of the study, participated in its design and coordination, performed most of the analysis, and drafted the manuscript. MG conceived of the study, participated in its design and coordination, and helped to draft the manuscript. EA participated in the data analysis, and helped to draft the manuscript. KY, MU and DW participated in its design, and helped to draft the manuscript. All authors read and approved the final manuscript.

\section{Acknowledgements}

We acknowledge support from the NIH and from the AL Williams Professorship funds. This work was also supported by the start-up funding package provided to CC by the Geisel School of Medicine at Dartmouth College.

\section{Author details}

'Department of Genetics, Geisel School of Medicine at Dartmouth, Hanover, New Hampshire, USA. ${ }^{2}$ Institute for Quantitative Biomedical Sciences, Geisel School of Medicine at Dartmouth, Lebanon, New Hampshire, USA. ${ }^{3}$ Norris Cotton Cancer Center, Geisel School of Medicine at Dartmouth, Lebanon, New Hampshire, USA. ${ }^{4}$ Program in Computational Biology and Bioinformatics, Yale University, 260 Whitney Avenue, New Haven, CT 06520, USA. ${ }^{5}$ Department of Molecular Biophysics and Biochemistry, Yale University, 260 Whitney Avenue, New Haven, CT 06520, USA. 'Department of Computer Science, Yale University, 260 Whitney Avenue, New Haven, CT 06520, USA.

Received: 25 November 2014 Accepted: 10 March 2015

Published online: 31 March 2015

\section{References}

1. Barabasi AL, Albert L, Jeong H, Bianconl G. Power-law distribution of the world wide web. Science. 2000;287:2115.

2. Albert $\mathrm{R}$, Jeong $\mathrm{H}$, Barabasi AL. Error and attack tolerance of complex networks. Nature. 2000;406:378-82.

3. Adamic LA, Glance N. The political blogosphere and the 2004 U.S. election: divided they blog. Proceedings of the 3rd international workshop on Link discovery. ACM 2005;36-43.

4. Jeong H, Tombor B, Albert R, Oltvai ZN, Barabasi AL. The large-scale organization of metabolic networks. Nature. 2000;407:651-4.

5. Jeong $H$, Mason SP, Barabasi AL, Oltvai ZN. Lethality and centrality in protein networks. Nature. 2001;411:41-2.

6. Dixon SJ, Costanzo M, Baryshnikova A, Andrews B, Boone C. Systematic mapping of genetic interaction networks. Annu Rev Genet. 2009;43:601-25.

7. Schwikowski B, Uetz P, Fields S. A network of protein-protein interactions in yeast. Nat Biotechnol. 2000;18:1257-61.

8. Tong AH, Lesage $G$, Bader GD, Ding H, Xu H, Xin X, et al. Global mapping of the yeast genetic interaction network. Science. 2004;303:808-13.

9. Lee TI, Rinaldi NJ, Robert F, Odom DT, Bar-Joseph Z, Gerber GK, et al. Transcriptional regulatory networks in Saccharomyces cerevisiae. Science. 2002;298:799-804.

10. Harbison CT, Gordon DB, Lee TI, Rinaldi NJ, Macisaac KD, Danford TW, et al, Transcriptional regulatory code of a eukaryotic genome. Nature. 2004:431:99-104

11. Ptacek J, Devgan G, Michaud G, Zhu H, Zhu X, Fasolo J, et al. Global analysis of protein phosphorylation in yeast. Nature. 2005;438:679-84.

12. Breitkreutz A, Choi H, Sharom JR, Boucher L, Neduva V, Larsen B, et al. A global protein kinase and phosphatase interaction network in yeast. Science. 2010;328:1043-6.

13. Bulyk ML. Discovering DNA, regulatory elements with bacteria. Nat Biotechnol. 2005;23:942-4.

14. Ouwerkerk PB, Meijer AH. Yeast one-hybrid screening for DNA-protein interactions. Curr Protoc Mol Biol. 2001, Chapter 12:Unit 12.12.

15. Ren B, Robert F, Wyrick JJ, Aparicio O, Jennings EG, Simon I, et al. Genomewide location and function of DNA binding proteins. Science. 2000;290:2306-9.

16. Johnson DS, Mortazavi A, Myers RM, Wold B. Genome-wide mapping of in vivo protein-DNA interactions. Science. 2007;316:1497-502.

17. Cheng C, Alexander R, Min R, Leng J, Yip KY, Rozowsky J, et al Understanding transcriptional regulation by integrative analysis of transcription factor binding data. Genome Res. 2012;22:1658-67.

18. Shou W, Verma R, Annan RS, Huddleston MJ, Chen SL, Carr SA, et al. Mapping phosphorylation sites in proteins by mass spectrometry. Methods Enzymol. 2002;351:279-96.

19. Bhardwaj N, Kim PM, Gerstein MB. Rewiring of transcriptional regulatory networks: hierarchy, rather than connectivity, better reflects the importance of regulators. Sci Signal. 2010;3:ra79. 
20. $\mathrm{Yu} \mathrm{H}$, Gerstein M. Genomic analysis of the hierarchical structure of regulatory networks. Proc Natl Acad Sci U S A. 2006;103:14724-31.

21. Jothi R, Balaji S, Wuster A, Grochow JA, Gsponer J, Przytycka TM, et al. Genomic analysis reveals a tight link between transcription factor dynamics and regulatory network architecture. Mol Syst Biol. 2009;5:294

22. Cheng C, Yan KK, Hwang W, Qian J, Bhardwaj N, Rozowsky J, et al. Construction and analysis of an integrated regulatory network derived from high-throughput sequencing data. PLoS Comput Biol. 2011;7, e1002190.

23. Gerstein MB, Lu ZJ, Van Nostrand EL, Cheng C, Arshinoff BI, Liu T, et al. Integrative analysis of the Caenorhabditis elegans genome by the modENCODE project. Science. 2010;330:1775-87.

24. Ma HW, Buer J, Zeng AP. Hierarchical structure and modules in the Escherichia coli transcriptional regulatory network revealed by a new top-down approach. BMC Bioinformatics. 2004;5:199.

25. Ma HW, Kumar B, Ditges U, Gunzer F, Buer J, Zeng AP. An extended transcriptional regulatory network of Escherichia coli and analysis of its hierarchical structure and network motifs. Nucleic Acids Res. 2004;32:6643-9.

26. Hartsperger ML, Strache R, Stumpflen V. HiNO: an approach for inferring hierarchical organization from regulatory networks. PLoS One. 2010;5, e13698.

27. Ispolatov I, Maslov S. Detection of the dominant direction of information flow and feedback links in densely interconnected regulatory networks. BMC Bioinformatics. 2008;9:424.

28. Sakata S, Yamamori T. Topological relationships between brain and social networks. Neural Netw. 2007;20:12-21.

29. Mok J, Zhu X, Snyder M. Dissecting phosphorylation networks: lessons learned from yeast. Expert Rev Proteomics. 2011:8:775-86.

30. Costanzo M, Baryshnikova A, Bellay J, Kim Y, Spear ED, Sevier CS, et al. The genetic landscape of a cell. Science. 2010;327:425-31.

31. Yan KK, Fang G, Bhardwaj N, Alexander RP, Gerstein M. Comparing genomes to computer operating systems in terms of the topology and evolution of their regulatory control networks. Proc Natl Acad Sci U S A. 2010;107:9186-91.

32. Krackhardt D. Graph theoretical dimensions of informal organizations. In: Carley KM, Prietula MJ, editors. Computational Organization Theory. Hillsdale, NJ: Lawrence Erlbaum and Associates; 1994. p. 89-111.

33. Mones $E$, Vicsek L, Vicsek T. Hierarchy measure for complex networks. PLoS One. 2012;7, e33799.

34. Corominas-Murtra B, Goni J, Sole RV, Rodriguez-Caso C. On the origins of hierarchy in complex networks. Proc Natl Acad Sci U S A. 2013;110:13316-21.

35. Roberts PJ, Der CJ. Targeting the Raf-MEK-ERK mitogen-activated protein kinase cascade for the treatment of cancer. Oncogene. 2007;26:3291-310.

36. Freschi L, Courcelles M, Thibault P, Michnick SW, Landry CR. Phosphorylation network rewiring by gene duplication. Mol Syst Biol. 2011;7:504.

37. Kirkpatrick S, Gelatt Jr CD, Vecchi MP. Optimization by simulated annealing Science. 1983;220:671-80

38. Newman RH, Hu J, Rho HS, Xie Z, Woodard C, Neiswinger J, et al. Construction of human activity-based phosphorylation networks. Mol Syst Biol. 2013:9:655.

39. Svetlov W, Cooper TG. Review: compilation and characteristics of dedicated transcription factors in Saccharomyces cerevisiae. Yeast. 1995;11:1439-84.

40. Horak CE, Luscombe NM, Qian J, Bertone P, Piccirrillo S, Gerstein M, et al. Complex transcriptional circuitry at the G1/S transition in Saccharomyces cerevisiae. Genes Dev. 2002;16:3017-33.

41. Borneman AR, Leigh-Bell JA, Yu H, Bertone P, Gerstein M, Snyder M. Target hub proteins serve as master regulators of development in yeast. Genes Dev. 2006;20:435-48

42. Borneman AR, Gianoulis TA, Zhang ZD, Yu H, Rozowsky J, Seringhaus MR, et al. Divergence of transcription factor binding sites across related yeast species. Science. 2007;317:815-9.

43. Consortium EP, Bernstein BE, Birney E, Dunham I, Green ED, Gunter C, et al. An integrated encyclopedia of DNA elements in the human genome. Nature. 2012;489:57-74.

44. Cheng C, Min R, Gerstein M. TIP: a probabilistic method for identifying transcription factor target genes from ChIP-seq binding profiles. Bioinformatics. 2011:27:3221-7.

45. Watts DJ, Strogatz SH. Collective dynamics of 'small-world' networks. Nature. 1998;393:440-2.

46. Ulanowicz RE, Heymans JJ, Egnotovich MS. Network analysis of trophic dynamics in South Florida ecosystems, FY 99: the graminoid ecosystem. Maryland System Center for Environmental Science. 2000: TS-191-99.
47. Leskovec J, Kleinberg J, Faloutsos C. Graph evolution: Densification and shrinking diameters. ACM Transactions on Knowledge Discovery from Data. 2007;1:2.

48. Cherry JM, Hong EL, Amundsen C, Balakrishnan R, Binkley G, Chan ET, et al. Saccharomyces Genome Database: the genomics resource of budding yeast. Nucleic Acids Res. 2012;40:D700-5.

49. Wall DP, Hirsh AE, Fraser HB, Kumm J, Giaever G, Eisen MB, et al. Functional genomic analysis of the rates of protein evolution. Proc Natl Acad Sci U S A. 2005; 102:5483-8.

50. Cherry JM, Ball C, Weng S, Juvik G, Schmidt R, Adler C, et al. Genetic and physical maps of Saccharomyces cerevisiae. Nature. 1997;387:67-73.

51. Winzeler EA, Shoemaker DD, Astromoff A, Liang H, Anderson K, Andre B, et al. Functional characterization of the $S$. cerevisiae genome by gene deletion and parallel analysis. Science. 1999;285:901-06.

52. Holstege FC, Jennings EG, Wyrick JJ, Lee TI, Hengarter $\mathrm{CH}$, Green MR, et al. Dissecting the regulatory circuitry of a eukaryotic genome. Cell. 1998;5:717-28.

53. Wang Y, Liu CL, Storey JD, Tibshirani RJ, Herschlag D, Brown PO. Precision and functional specificity in mRNA decay. Proc Natl Acad Sci U S A. 2002;99:5860-5.

54. Belle A, Tanay A, Bitincka L, Shamir R, O'Shea EK. Quantification of protein half-lives in the budding yeast proteome. Proc Natl Acad Sci U S A. 2006:103:13004-9.

55. Newman JR, Ghaemmaghami S, Ihmels J, Breslow DK, Noble M, DeRisi JL, et al. Single-cell proteomic analysis of $\mathrm{S}$. cerevisiae reveals the architecture of biological noise. Nature. 2006;441:840-6.

56. Huh WK, Falvo JV, Gerke LC, Carroll AS, Howson RW, Weissman JS, et al. Global analysis of protein localization in budding yeast. Nature. 2003:425:686-91.

57. da Huang W, Sherman BT, Lempicki RA. Systematic and integrative analysis of large gene lists using DAVID bioinformatics resources. Nat Protoc. 2009:4:44-57.

58. Ripeanu M, lamnitchi A, Foster IT. Mapping the Gnutella Network. IEEE Internet Comput. 2002;6:50-7.

\section{Submit your next manuscript to BioMed Central and take full advantage of:}

- Convenient online submission

- Thorough peer review

- No space constraints or color figure charges

- Immediate publication on acceptance

- Inclusion in PubMed, CAS, Scopus and Google Scholar

- Research which is freely available for redistribution 